\title{
Miniaturized EI/Q/oa TOF Mass Spectrometer
}

\author{
V. D. Berkout and R. J. Cotter \\ The Johns Hopkins University School of Medicine, Baltimore, Maryland, USA \\ D. P. Segers \\ O. I. Analytical, CMS Field Products, Pelham, Alabama, USA
}

\begin{abstract}
A miniaturized orthogonal time-of-flight mass spectrometer with an electron impact ionization ion source and a rf quadrupole ion guide has been developed. A mass resolving power of $\mathrm{m} / \Delta \mathrm{m}=5500$ has been obtained in a $0.4 \mathrm{~m}$ instrument. The addition of helium at pressures of about 4.0 mtorr into the ion source showed collisional focusing taking place in the rf quadrupole. An automated gas chromatograph designed for air monitoring applications has been coupled to the time-of-flight mass analyzer and tested for the detection of simulants of chemical-warfare agents. (J Am Soc Mass Spectrom 2001, 12, 641-647) (c) 2001 American Society for Mass Spectrometry
\end{abstract}

A dvanced easily portable chemical sensor systems are essential for the on-site analytical measurements required for a variety of diverse applications such as environmental characterization and monitoring, law enforcement, and chemical emergency response. For such applications, mass spectrometry-based systems that combine laboratory performance with high portability and robustness are required. Time-of-flight mass spectrometers (TOF-MS) look promising for realization of these goals because of their simple designs and rapid delivery of full mass spectra. However, the fact that limited mass resolution $\mathrm{m} / \Delta \mathrm{m}$ (up to a few hundred) has been obtained with electron impact ionization has limited their applications for the analysis of volatile and semi-volatile chemical compounds.

The major limitation comes from the initial velocity distribution of ions created by electron impact in the storage ion source [1], and particularly the inability to compensate for the turn-around time described early on by Wiley and McLaren [2]. The mass resolution of the TOF can be increased by the addition of an ion mirror [3]. An ion mirror images the McLaren space-time focus plane to the plane of the ion detector in such a manner that ions with differing energies (but the same mass) will arrive at the detector at the same time. The resolution of this type of instrument, when coupled to a gas chromatograph, is still limited because of a continuous gas load to the ion source located in the analyzer chamber. With this technology a mass resolution of

Address reprint requests to Dr. R. J. Cotter, Department of Pharmacology and Molecular Science, Middle Atlantic Mass Spectrometry Laboratory, Johns Hopkins University School of Medicine, 725 N. Wolfe Street, Baltimore, MD 21205. E-mail: rcotter@jhmi.edu about 700 was obtained on a $1.5 \mathrm{~m}$ long TOF instrument [4].

An alternative approach consists in forming the ion beam in a direction which is perpendicular to the time-of-flight direction. The original velocity spread in the longitudinal direction no longer affects the arrival time of an ion packet on the detector. An orthogonal injection TOF was first proposed in 1964 [5] but has been reintroduced as a method for improving mass resolution [6, 7]. A mass resolution of $\mathrm{m} / \Delta \mathrm{m}>10,000$ was demonstrated recently in a $1.2 \mathrm{~m}$ long instrument with an orthogonal electron impact ion source and ion mirror [8].

In this paper we present a compact time-of-flight mass spectrometer with an orthogonal electron impact ion source. An rf quadrupole used as an ion focusing element allowed us to achieve high mass resolving power at elevated pressures in the ion source housing. To demonstrate the potential of orthogonal-extraction, time-of-flight mass spectrometer for air-monitoring applications, a commercially available automated gaschromatographic monitoring system was interfaced to the mass spectrometer, and preliminary analyses were performed.

\section{Experimental}

The time-of-flight mass spectrometer with orthogonal acceleration and an electron impact ion source is shown schematically in Figure 1. It consists of ion optics for the formation and focusing of a low energy $(10-20 \mathrm{eV})$ ion beam, which moves in the longitudinal direction, and orthogonal acceleration optics that accelerates these ions in the time-of-flight direction to $\sim 2300 \mathrm{eV}$.

The compounds to be analyzed are introduced into 


\section{$0.36 \mathrm{~m}$}

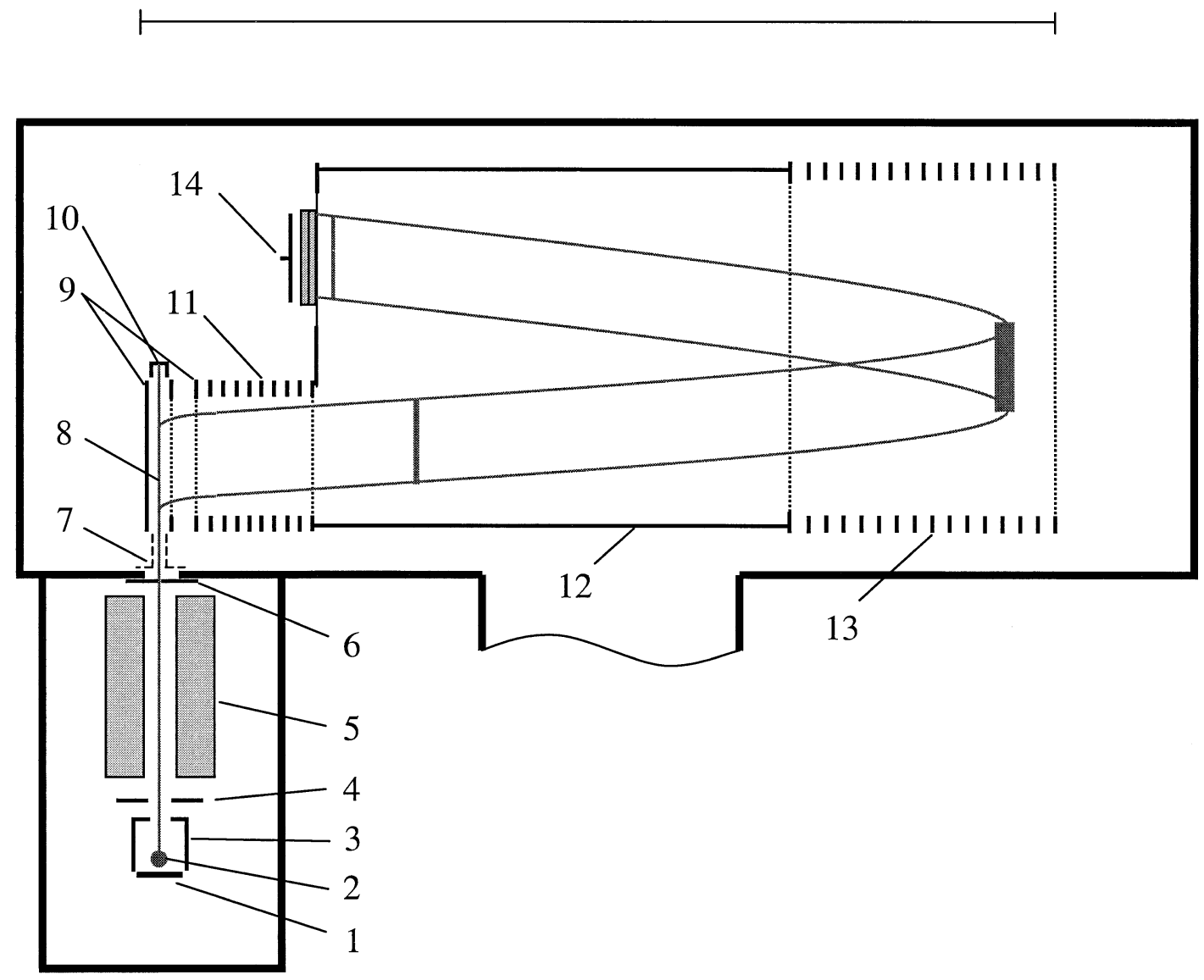

Figure 1. Schematic diagram of the time-of-flight mass spectrometer with orthogonal acceleration and electron impact ion source.

the ion source 3 via a Granville-Phillips (Boulder, CO) variable leak valve. The ion source is floating at some positive potential, (typically $8-12 \mathrm{~V}$ ), to provide the necessary velocity in the longitudinal direction. Ions are produced by interaction with an electron beam 2 of about $70 \mathrm{eV}$. Electrons were produced continuously by an electrically heated tungsten filament mounted on a ceramic base (Kimball Physics, Wilton, NH). The electron beam was focused by a pierce element and a single lens into the ion source through a 1.0 -mm-diameter aperture. The electron current measured by a trap located behind the ion source was about $18 \mu \mathrm{A}$. A magnetic field created by a pair of 0.5 -inch-diameter SmCo magnets along the electron beam caused the electrons to follow a helical path, thereby increasing the probability of interaction with sample molecules. Ions are then pushed by a repeller 1 and focused by lens 4 at the entrance of a quadrupole 5 . The quadrupole ion guide focuses ions on a small $(0.5 \mathrm{~mm}$ diameter) aperture 6, which separates the ion source housing and the time-of-flight analyzer. The time-of-flight analyzer chamber is pumped by a Leybold (Export, PA) Model Turbovac 361350 l/sec turbopump. The quadrupole rods $(0.95 \mathrm{~cm}$ diameter and $7.6 \mathrm{~cm}$ long) are supported by machinable alumina ceramic holders whose surfaces are screened from the ion beam. The quadrupole is driven by a small SRS (Sunnyvale, CA) Model DS340 sine-wave signal generator coupled through an ENI (Rochester, NY) Model 240L broadband rf power amplifier which exhibits flat gain over a wide range of operating frequencies. A rf coupling transformer built in-house gives an output voltage from 0 to $1000 \mathrm{~V}$ peak-to-peak in the frequency range from $100 \mathrm{kHz}$ to 5 $\mathrm{MHz}$. The transformer also provides the required $180^{\circ}$ phase difference between the rod pairs. The quadrupole rods are offset to some dc potential applied through decoupling capacitors. The dc voltages applied to the lens and aperture plate, as well as to the quadrupole, are adjusted to obtain optimum ion transmission.

After passing through the aperture ions go into the storage region 8 of the time-of-flight mass analyzer. Deflection plates 7 shield the low energy ion beam from high voltages applied to the accelerator column 11, and also make a final adjustment of the beam. An ion collector 10, located right after the storage region, measures the intensity of the ion beam. Initially, the electrical field in the gap of the storage region is close to zero. The last grid of the extraction plates 9 is held at a 


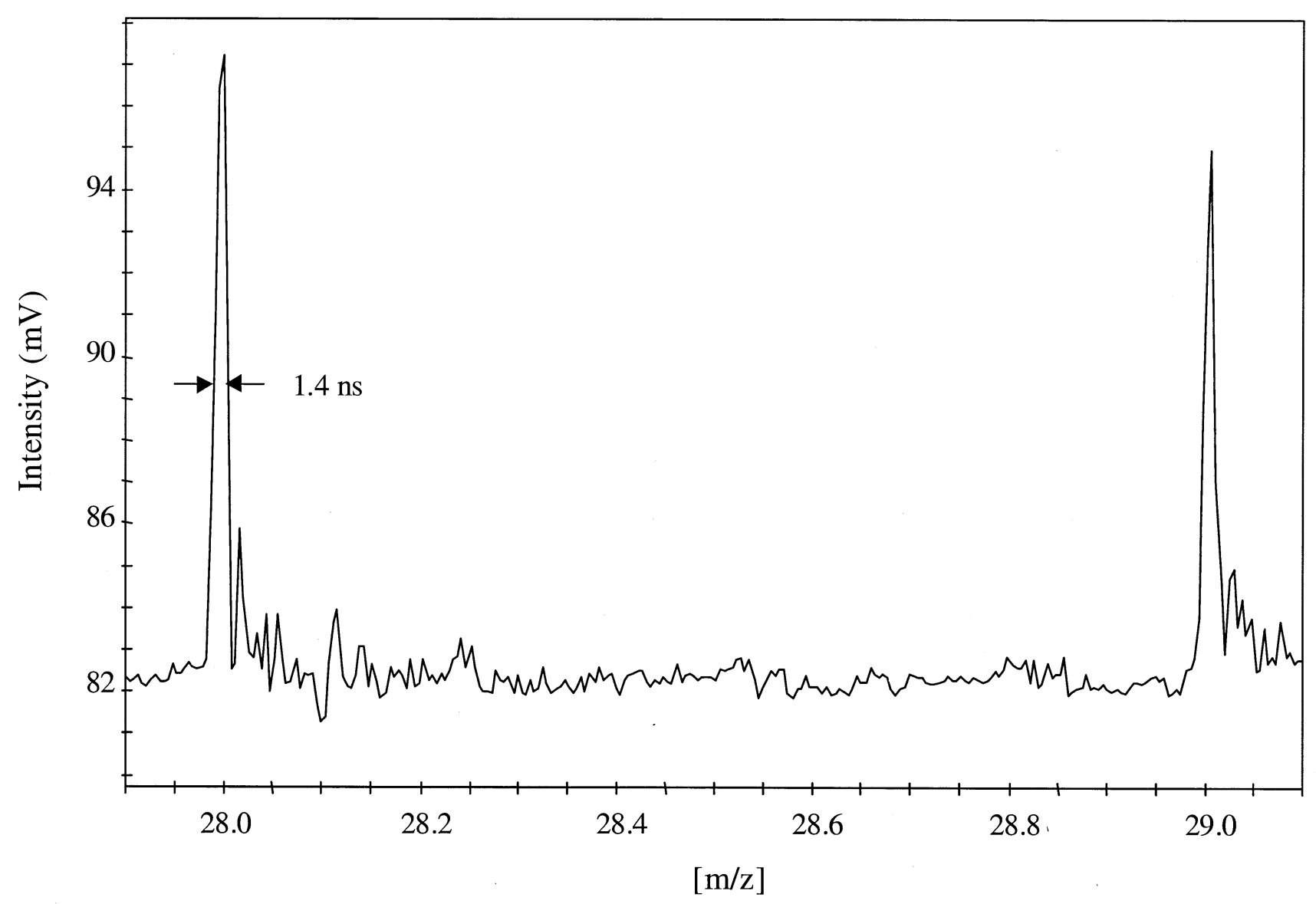

Figure 2. Mass resolution in the low mass range.

small positive potential (typically $6-12 \mathrm{~V}$ ) to prevent field penetration [7] from the accelerator column into the storage region.

When the storage region is filled with ions, both push-out and draw-out voltage pulses are applied simultaneously to extraction plates. The intermediate plate is kept at ground potential. Extraction pulses, produced by Eurotek (Morganville, NJ) Model HTS 31GSM high voltage push-pull switches, have typical amplitudes of +300 and $-300 \mathrm{~V}$, a risetime of $\sim 30 \mathrm{~ns}$, and a duration of $\sim 2 \mu \mathrm{s}$. The duration of the pulse is chosen to guarantee that the heaviest ions have enough time to leave the storage region. The values of the draw-out and push-out pulses are chosen to ensure a homogeneous electric field between extraction plates. This reduces ion scattering near the intermediate grid where defocusing effects could be strong for ions with a relatively low kinetic energy.

After leaving the storage region, ions are accelerated to energies of a few $\mathrm{keV}$ by a uniform dc field in the acceleration column. The ions then move with constant velocities in the field free drift region 12. The lengths of the storage, acceleration and field free regions are 1.2 $\mathrm{cm}, 5.2 \mathrm{~cm}$ and $18.7 \mathrm{~cm}$, respectively. In order to keep the ion source and ion storage potentials close to the ground, the drift region is floated at high voltage potential. A perforated metal cover prevents penetration of the ground potential of the vacuum chamber into the drift region, while allowing effective pumping of the inside volume. Having traversed the drift region, ions enter a single-stage electrostatic mirror 13, which provides first-order correction for the kinetic energy spread [3]. The field free region is separated from the accelerator column and electrostatic mirror by Buckbee Mears (St.Paul, MN) grids with a transmission of $88.6 \%$.

Reflected ions pass back through the drift region before striking the detector 14. The detector is a Hamamatsu (Bridgewater, NJ) Model F4655-13 dual microchannel plate assembly with conical anode and 50 $\Omega$ impedance matching. The small channel diameter (4 $\mu \mathrm{m})$ and $\mathrm{a} 12^{\circ}$ bias angle allows the detector to record the ion peaks with temporal resolution better than 0.5 ns. The front plate of the detector is at the same potential as the drift region $(-2.3 \mathrm{kV})$, thus keeping the anode at ground potential. Voltages for the pulsers, drift region and ion mirror were provided by Bertan (Hicksville, NY) Models 301 and 325 stable, low noise, high voltage power supplies.

The electron impact ion source implemented on this instrument provides high ionization efficiency and ion transmission. At pressures of residual air of $10^{-5}$ torr in the ion source chamber, the ion current on the ion 


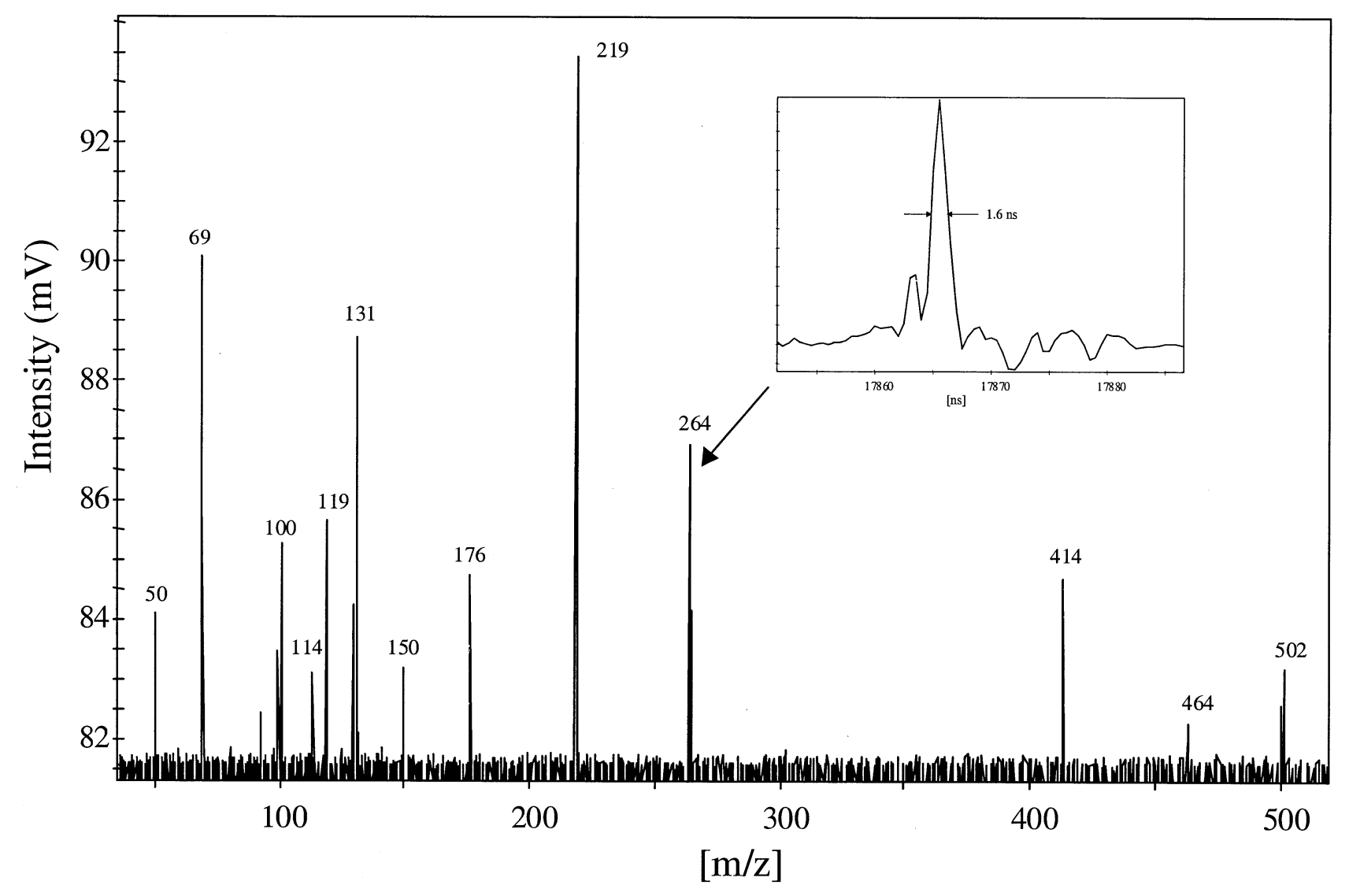

Figure 3. EI spectra of perfluorotributylamine recorded on the miniaturized oa TOF mass spectrometer.

collector located behind the extraction plates was about $1 \mathrm{pA}$. Since the number of ions of the same mass in the ion packet is expected to be more than one per extraction pulse, an Ortec (Oak Ridge, TN) FastFlight integrated transient recorder is used to record the spectra. The trigger from the FastFlight recorder starts the extraction pulses in the time-of-flight mass spectrometer. In order to sample the repetitive signal at exactly the same points in each scan, triggering is highly synchronized (jitter less than $50 \mathrm{ps)} \mathrm{with} \mathrm{the} \mathrm{sampling} \mathrm{clock} \mathrm{in}$ the FastFlight system.

\section{Results and Discussion}

\section{Instrument performance}

The performance of the compact time-of-flight mass spectrometer with orthogonal extraction was first checked by recording the spectra of residual air in the ion source chamber. The rf quadrupole ion guide was operated at a frequency of $1.75 \mathrm{MHz}$ and voltage amplitude of $120 \mathrm{~V}$. The expanded part of the low mass range is shown in Figure 2. It represents the average of 200 spectra obtained at an effective 2-GHz sampling rate from the 2 ns analogto-digital converter (ADC) in the interleaving mode that increments the delay between the extraction pulse trigger and the sampling clock by $0.5 \mathrm{~ns}$ in four successive scans. Using a repetition rate of $4 \mathrm{kHz}$, the entire spectrum, shown in Figure 2, was acquired in $0.05 \mathrm{~s}$. The peak width of $1.4 \mathrm{~ns}$ full-width-at-half-maximum (FWHM) corresponds to a mass resolution $(\mathrm{m} / \Delta \mathrm{m}=\mathrm{t} / 2 \Delta \mathrm{t})$ of about 1600 for $m / z=28$.

To test the instrument performance at higher ion masses, perfluorotributylamine PFTBA, commonly known as the calibration standard FC43, was introduced into the ion source through a variable leak valve. PFTBA was obtained from Scientific Instrument Services (Ringoes, NJ). The average of 200 spectra with a 4 $\mathrm{kHz}$ repetition rate is shown in Figure 3. The mass spectra in the $m / z>100$ range corresponds well to the library spectra [9]. In the low mass range some mass discrimination occurs. For example, the intensity of the $\mathrm{CF}_{3}^{+}$ion $(m / z=69)$ is decreased about three times. This low mass discrimination probably results from a decreased transmission of light ions through the quadrupole ion guide and aperture optics.

The peak width of 1.6 ns FWHM for $m / z=264$ corresponds to mass resolution of 5500 at this mass. The mass resolution increases significantly compared with that observed for $m / z=28$ because the flight time has increased from $4.7 \mu$ s to $17.8 \mu$ s and the peak width has 


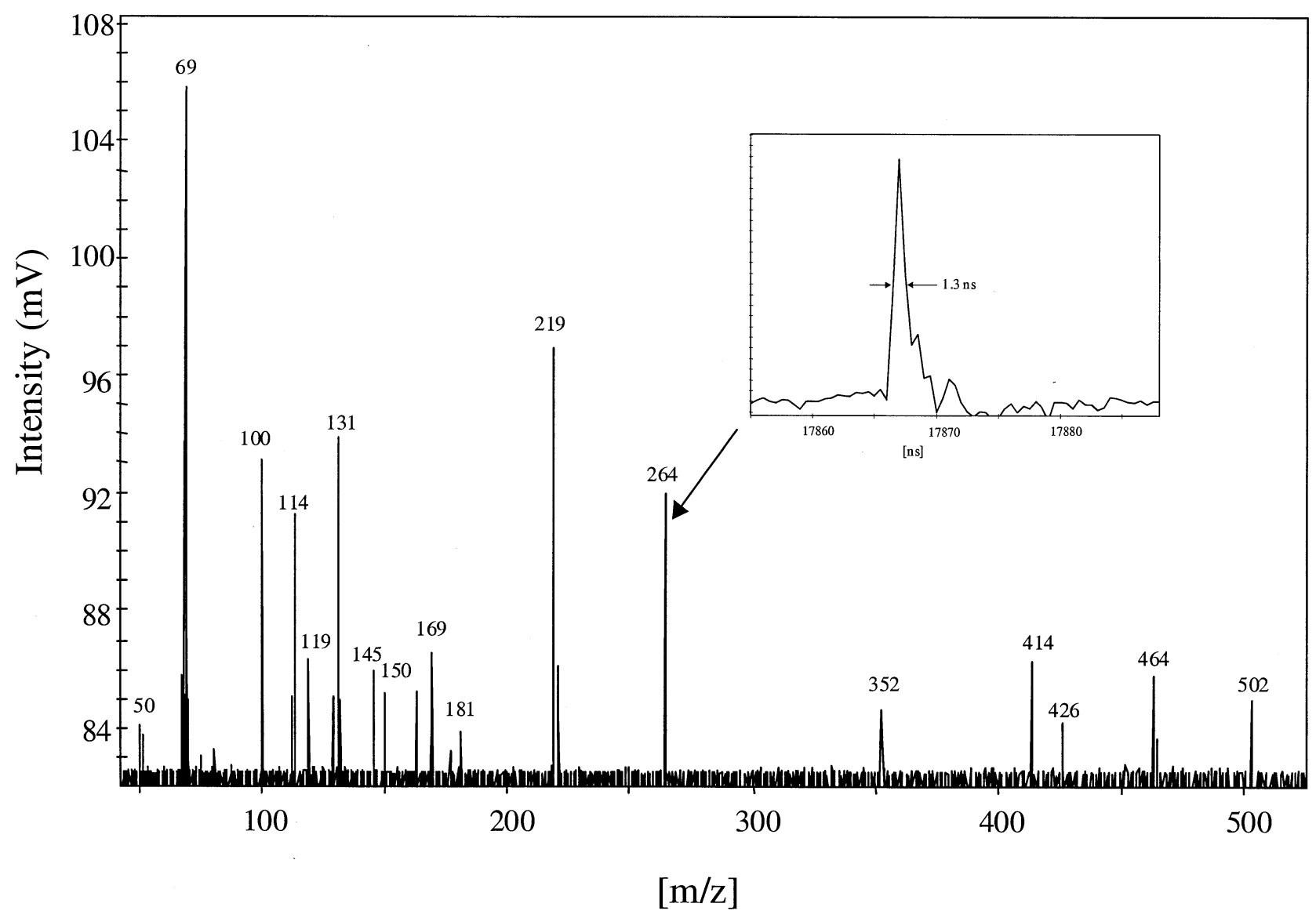

Figure 4. EI spectra of perfluorotributylamine with helium pressure of $4.0 \mathrm{mTorr}$ in the ion source.

increased by only by $14 \%$. The small increase in the ion peak widths for higher masses indicates [10] that the major factors limiting a mass resolution in our system come from detector response and electronics. The finite pulse width produced by an incoming ion on the detector broadens further because of limitations of the electronics, which processes and averages this signal. Small variations in the electronics providing repetitive high voltage extraction pulses could also add to peak broadening.

To model the conditions of sample introduction through a gas chromatograph, helium was introduced into the ion source housing. The pressure was measured by a Leybold Inficon (Export, PA) Model CR090 capacitance diaphragm gauge. The results for a helium pressure of 4.0 mtorr are presented in Figure 4. The intensities of all ions, especially in the low mass range, have increased. In particular, the intensity of the $\mathrm{m} / \mathrm{z}=69$ peak has increased more than three times. Mass resolution, as illustrated for $m / z 264$, has also increased. This indicates that collisional cooling is occurring in the quadrupole ion guide [11, 12]. Subsequent addition of more helium led to a decrease in intensities of all ions. At 7.0 mtorr of $\mathrm{He}$, ion intensities were comparable to vacuum conditions, and at higher pressures lower intensity ions began to disappear.
Preliminary results for air monitoring applications.

To demonstrate that the time-of-flight mass spectrometer could potentially be used for air-monitoring applications, an OI Analytical/CMS Field Products (Pelham, AL) MINICAMS unit was interfaced to the mass spectrometer. The MINICAMS is an automated system designed for air monitoring applications, including low-level monitoring for toxic compounds. The MINICAMS, with a solid-sorbent sampling inlet, was modified by replacing the standard detector with a heated transfer block and a heated transfer line to transfer analyte from the MINICAMS to the mass spectrometer. The heated transfer line contained deactivated silica capillary tubing to transfer compounds, as they eluted from the gas-chromatographic column, into the ionization chamber of the mass spectrometer. The ionization chamber was additionally pumped by a Balzers (Hudson, NH) Model TPH050 50 l/s turbopump. The aperture size, which separates the ion source housing and the time-of-flight analyzer, was increased to $1.0 \mathrm{~mm}$ diameter.

The MINICAMS automatically cycles between a sampling period and a purge, (or analytical), period. During the sampling period, air is drawn (by vacuum) into the instrument through a preconcentrator tube 


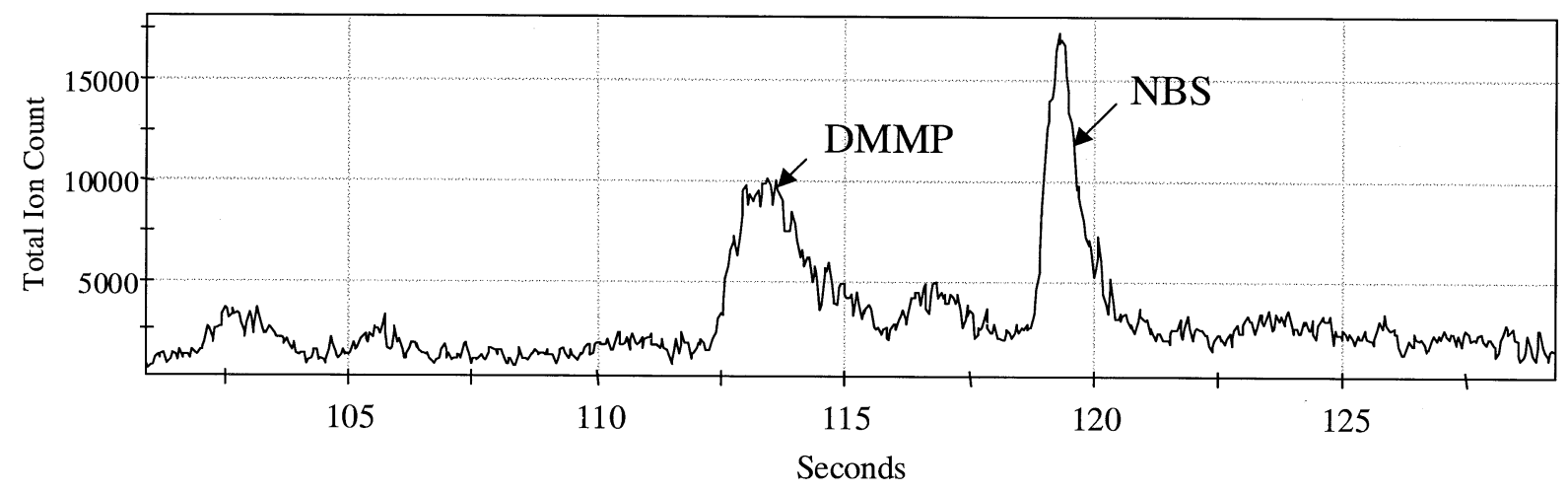

DMMP

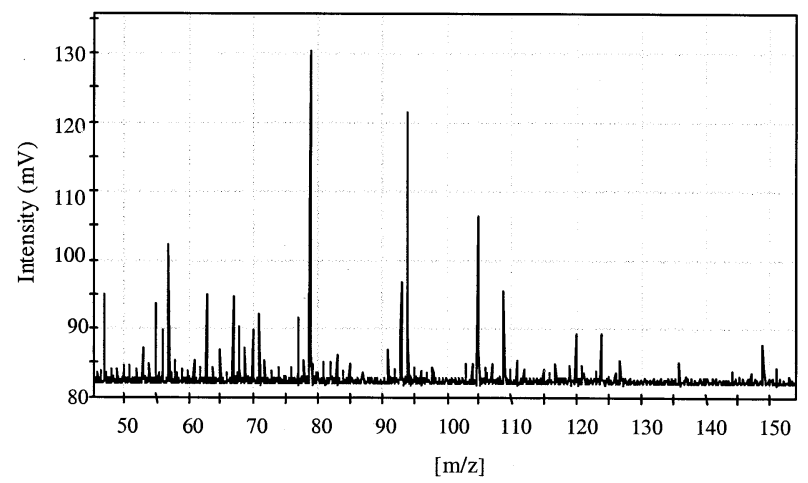

NBS

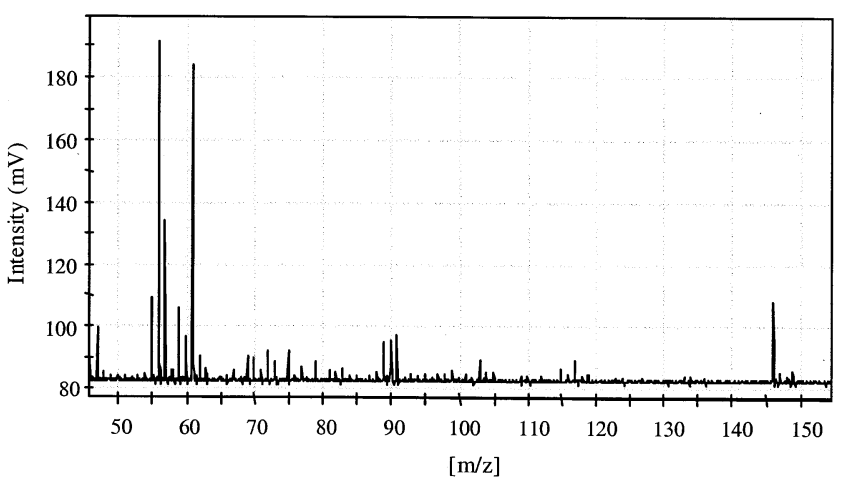

Figure 5. In-injection port thermal desorption MINICAMS/TOF chromatogram of DMMP (20 ng) and NBS (10 ng) and corresponding mass spectra.

containing a solid sorbent to collect and preconcentrate volatile and/or semi-volatile compounds from the sampled air. At the end of the sampling period the preconcentrator tube is backflushed with the carrier gas (nitrogen or helium) while it is heated to desorb the analytes onto the front of a gas-chromatographic column. After desorption onto the column, the column is heated to separate the collected analytes before reaching the detector, which in this case was the time-offlight mass analyzer.

Figure 5 shows a representative total-ion chromatogram obtained with the MINICAMS interfaced to the time-of-flight mass spectrometer. The chromatogram was obtained by injecting standards of dimethyl methylphosphonate (DMMP) and n-butyl sulfide (NBS) into the MINICAMS inlet during the sampling period to simulate the collection of vapors during air sampling. A dual-bed solid-sorbent preconcentrator tube was used. DMMP and NBS were selected as simulants for the chemical-warfare agents sarin and mustard, respectively. The total-ion chromatogram shows the chromatographic peaks obtained for DMMP (retention time of $113 \mathrm{~s}$ ) and NBS (retention time of $119 \mathrm{~s}$ ) as well as a few background peaks. Also shown in Figure 5 are the average of 400 mass spectra for DMMP and NBS recorded by time-of-flight mass spectrometer in $0.1 \mathrm{~s}$.

The MINICAMS configuration and operating parame- ters were selected to analyze DMMP and NBS but were also selected for compatibility with the time-of-flight mass-spectrometer. In particular the flow split of approximately 1:10 was used to reduce the gas load to a mass spectrometer. The injection amounts for DMMP (20 ng) and NBS (10 ng) were the in-inlet port amounts; however, the analyte reaching the mass spectrometer was less than the in-inlet port amount because of incomplete recovery from sorbent material and because the carrier flow was split after it eluted from the column. The carrier gas was helium, and the flow rate to the mass spectrometer was approximately $1 \mathrm{~mL} / \mathrm{min}$.

\section{Conclusions and Future Prospects}

The miniaturized orthogonal time-of-flight mass spectrometer with an electron impact ionization ion source and a rf ion guide produces full mass spectra in $0.05 \mathrm{~s}$ with a mass resolution of $\sim 5500 \mathrm{FWHM}$ at $m / z 264$. The ability to rapidly identify simulants of chemical-warfare agents was demonstrated when coupled to an air monitoring system. Limits of detection of simulants for several chemical-warfare agents were found to be between 3 and $10 \mathrm{ng}$. Future development efforts will focus on reducing the total carrier flow rate and removing the split to increase overall system sensitivity. 


\section{Acknowledgments}

This work was supported in part by a subcontract from the Johns Hopkins University Applied Physics Laboratory and funded by the Defense Advanced Research Projects Agency (DARPA).

\section{References}

1. Grix, R.; Grüner, U.; Li, G.; Stroh, H.; and Wollnik, H. Int. J. Mass Spectrom. Ion Proc. 1989, 93, 323.

2. Wiley, W. C.; McLaren, I. H. Rev.Sci.Instrum. 1955, 26, 1150.

3. Mamyrin, B. A.; Karataev, D. V.; Shmikk, D. V.; Zagulin, V. A. Sov. Phys. JETP 1973. 37, 45.

4. Wollnik, H.; Becker, R.; Götz, H.; Kraft, A.; Jung, H.; Chen, C.-C.; Van Ysacker, P. G.; Janssen, H.-G.; Snijders, H. M. J.; Leclercq, P. A.; Cramers, C. A. Int. J. Mass Spectrom. Ion Proc. 1994, L7, 130.
5. O'Halloran, G. J.; Fluegge, R. A.; Betts, J. F.; Everett, W. L. Rep. ASD-TDR 62-644, Bendix Co. Res. Lab., 1964.

6. Dawson, J. H. J.; Guilhaus, M. Rapid Commun. Mass Spectr. 1989, 3, 155.

7. Dodonov, A. F.; Chernushevich, I. V.; Laiko, V. V. In Time-ofFlight Mass Spectrometry, Cotter, R. J. Ed.; American Chemical Society, 1994; p 108.

8. Chen, Y. H.; Gonin, M.; Fuhrer, K.; Dodonov, A.; Su, C. S.; Wollnik, H. Int. J. Mass Spectrom. Ion Proc. 1999, 221, 185-187.

9. NIST98 Mass Spectral Library.

10. Coles, J. N.; Guilhaus, M. J.Am.Soc. Mass Spectrom.1994, 5, 772.

11. Douglas, D. J.; French, J. B. J.Am.Soc. Mass Spectrom. 1992, 3, 398.

12. Kozlovsky, V.; Fuhrer, K.; Tolmachev, A.; Dodonov, A.; Raznikov, V.; Wollnik, H. Int. J. Mass Spectrom. Ion Proc. 1998, 27, 181. 